ИЗВЕСТИЯ АКАДЕМИИ НАУК ЭСТОНСКОИ ССР. ТОМ 26 ХИМИЯ * ГЕОЛОГИЯ. 1977, № 4

\title{
ТЕРМОЛЮМИНЕСЦЕНТНЫЕ И ДОЗИМЕТРИЧЕСКИЕ СВОЙСТВА КВАРЦА ИЗ ЧЕТВЕРТИЧНЫХ ОТЛОЖЕНИЙ
}

Определение физического возраста отложений относится к числу наиболее сложных проблем современной геологии. Широко используемые в настоящее время методы датирования - радиоуглеродный, калий-аргоновый, палеомагнитный и другие - имеют ряд ограничений, поэтому поиски новых методов находятся в центре внимания геологов. В последние годы при определении возраста разногенетических четвертичных отложений стал применяться термолюминесцентный (ТЛ) метод датирования (Шелкопляс, 1974). Недостатки методики подробно проанализированы нами ранее (Хютт, Раукас, 1977). Заметим лишь; что отсутствие разработки физической стороны проблемы и ряд прямых ошибок ставят под сомнение степень достоверности полученных датировок.

За последнее десятилетие разработан ряд оригинальных методик датирования в археологии (Aitken и др., 1968; Fleming, 1970; Fleming, Stoneham, 1971), основанных на результатах тщательного исследования дозиметрических свойств кварца, экстрагированного из древней керамики, с целью использования его для ТЛ датирования во временном интервале $1-8$ тыс. лет.

В настоящем сообщении изучаются возможности применения кварца для датирования осадочных отложений, возраст которых охватывает интервал $10^{3}-10^{6}$ лет. Расширение временного интервала и специфика геологического объекта потребовали от нас решения следующих принципиальных задач.

1. Исследовать ТЛ свойства кварца из разногенетических четвертичных отложений.

2. Исследовать дозиметрические особенности кварца для выяснения возможности использовать его в качестве дозиметра для столь широких временных интервалов.

3. Попытка доказать существование «нуль-момента» в кварце осадочных отложений, т. е. стирание прогенетической светосуммы минерала, - проблемы, не существующей для керамики, так как там «нульмомент» автоматически обеспечивается последним отжигом изделия.

Минеральные включения в отложениях (кварц и полевой шпат) по工 влиянием радиоактивных примесей окружения (U, Th, ${ }^{40} \mathrm{~K}$ ) возбуждаются и аккумулируют энергию в виде запасенной светосуммы. Основной принцип ТЛ датирования (Даниельс и др., 1953) состоит в том, что аккумулированная доза, информация о которой получается сравнением естественной ТЛ (ЕТЛ) кварца с индуцированной ТЛ (ИТЛ) от источника радиации известной мощности, делится на годичную дозу, оценку 
которой получают по общему содержанию радиоактивных примесей. Частное от деления и составляет возраст. Хотя принцип весьма прост, методика ТЛ датирования сложна и требует учета многих факторов. В настоящем сообщении мы не будем останавливаться на вариантах конкретной методики датирования, а лишь попытаемся оценить возможность использования кварца в качестве возрастной ячейки памяти четвертичных отложений.

\section{Способ экстрагирования кварца из осадочных отложений и методика исследования}

Поскольку люминесценция является свойством не валовой пробы, а лишь определенного минерала, перед нами стояла задача довольно тщательного выделения мономинеральной фракции.

Методом мокрого ситового анализа из образца выделялась фракция крупностью 100-140 мкм. Гранулометрический диапазон выбора определило относительно большое содержание зерен именно такого размера в исследуемых объектах. Кварц из пробы экстрагировался методом минеральной сепарации в тяжелых жидкостях. Как известно, удельный вес кварца 2,63-2,67 г/см ${ }^{3}$. Нами использовались растворы бромоформа и ксилола, имеющие плотности 2,68 и 2,62 г/c ${ }^{3}$ соответственно. Первой жидкостью выделялась фракция тяжелых минералов с $D=2,68$ г/c.⿲丿丨 ${ }^{3}$,

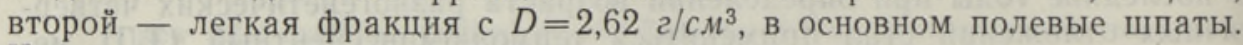
Качество экстрагирования контролировалось под микроскопом, для исследования использовались образцы кварца со степенью чистоты 98-99\%. Очистка поверхности зерен кварца от загрязнений производилась обработкой в смеси $\mathrm{HF}+\mathrm{HCl}$ (по 30 мл) $+\mathrm{H}_{2} \mathrm{O}(300$ мл) в течение 10 мин, либо ультразвуком на установке типа УЗДН-1 в течение 2-3 мин. Определенность гранулометрического состава и чистота поверхности зерен гарантировали постоянство световыхода при облучении стандартной дозой и хорошую воспроизводимость результатов. Рентгенолюминесценция кварца изучалась на установке, смонтированной из спектрофотометра СФ-4, рентгеновского аппарата УРС-1,0 и криостата в диапазоне температур $90-670 \mathrm{~K}$. Используемая нами конструкция обеспечивала сканирование в оптическом интервале 200-1100 нм. Спектральные и температурные отметки в процессе работы наносились автоматически. Естественная и индуцированная термолюминесценции исследовались в зеркальном криостате специальной конструкции, что давало возможность производить измерения в вакууме, воздухе и азоте высокой степени чистоты (кислорода не более $0,001 \%$, влаги не более $\left.0,0052 / \mathrm{M}^{3}\right)$. Специально разработанная система равномерного нагрева обеспечивала скорость процесса 0,25 град/сек, температура записывалась автоматически.

На обеих установках сигналы регистрировались с помощью фотоэлектронных умножителей, усилителей постоянного тока и автоматических самописцев типа ЭПП-09М либо КСП-4. Источниками возбуждения служили рентгеновская трубка с Сu-анодом и $\gamma$-источники ${ }^{60} \mathrm{Co}$, мощности экспозиционных доз составляли 1,5 и 0,15 p/ceк.

\section{Структура дефектов кристаллической решетки и люминесценция кварца}

Основой кристаллической структуры кварца является гексагональная решетка. Элементарная ячейка $\mathrm{Si}_{3} \mathrm{O}_{6}$ имеет размеры $a_{0}=4,913 \AA$, $c_{0}=5,405 \AA$ (Дэна и др., 1966). Структура кварца сложена тетраэдрами 
$\mathrm{SiO}_{4}$ : каждый атом кислорода связан с двумя атомами $\mathrm{Si}$, т. е. атомы в вершинах одного тетраэдра принадлежат и другим тетраэдрам.

Наиболее часто встречающейся изоморфной примесью кварца является $\mathrm{Al}^{3+} \rightarrow \mathrm{Si}^{4+}$, компенсация валентности осуществляется одно- и двухвалентными катионами: $\mathrm{Li}^{+}, \mathrm{Na}^{+}, \mathrm{H}^{+}, \mathrm{Mg}^{2+}, \mathrm{Fe}^{2+}, \mathrm{Mn}^{2+}, \mathrm{Ca}^{2+}\left(\mathrm{Kaц,}^{2}\right.$ Симанович, 1974).

В исследовании структуры примесных и собственных дефектов кварца, особенно синтетического, достигнуты значительные успехи (Батрак, 1958 ; Физические исследования кварца, 1975). Установлено, что наиболее стабильным центром свечения является тетраэдрический комплекс $\mathrm{AlO}_{4}^{4-}\left(E_{\text {нзл }}=2,65-2,7\right.$ э $\left.B\right)$. Он проявляется в рентгенолюминесценции, фотолюминесценции и является центром рекомбинации в процессе ТЛ. Есть также четыре типа парамагнитных центров, обусловленных собственными дефектами кварца, два из которых имеют өлектронную природу $-E_{1}$ и $E_{2}$, а два - дырочную $-E_{3}$ и $E_{4}$. Центры $E$-типа проявляются при облучении природного кварца электронами. Известны также ЭПР-центры примесного характера, связанные с Al, Ge, Тi. Для ряда ЭПР-центров осуществлена корреляция с отдельными полосами спектров поглощения кварца. Существенные успехи достигнуты в изучении типов электронных возбуждений синтетического кварца (Закис и др., 1973), а также в исследовании энергетического спектра, обусловливающего появление максимумов ТЛ в низкотемпературной области (Мендзиня, Тале, 1974).

Что касается природы уровней захвата, связанных с высокотемпературными максимумами кривой ТЛ, то литературных данных пока немного. Заслуживают внимания соображения (Физические исследования кварца, 1975) о связи глубоких уровней захвата синтетического кварца $\left(T_{M}=\right.$ $=570 \mathrm{~K})$ с примесями $\mathrm{Al}$ и $\mathrm{Na}$, в крнсталлах с примесью $\mathrm{Ge}$ фиксируется также пик ТЛ с $T_{M}=$ $=445 \mathrm{~K}$ (скорость нагрева 0,2 град/сек). Конкретная структура центров захвата, к сожалению, не была обсуждена.

Рис. $1, a-$ ТЛ свойства кварца: при рентгеновском возбуждении (I), при облучении $\gamma$-источником (2) и ЕТЛ (3); 6 - спектральный состав ТЛ; $\boldsymbol{8}$ - влияние состава атмосферы прогрева на величнну ТЛ кварца: фоновое свечение $(I)$, прогрев в азоте (2) и воздухе (3).

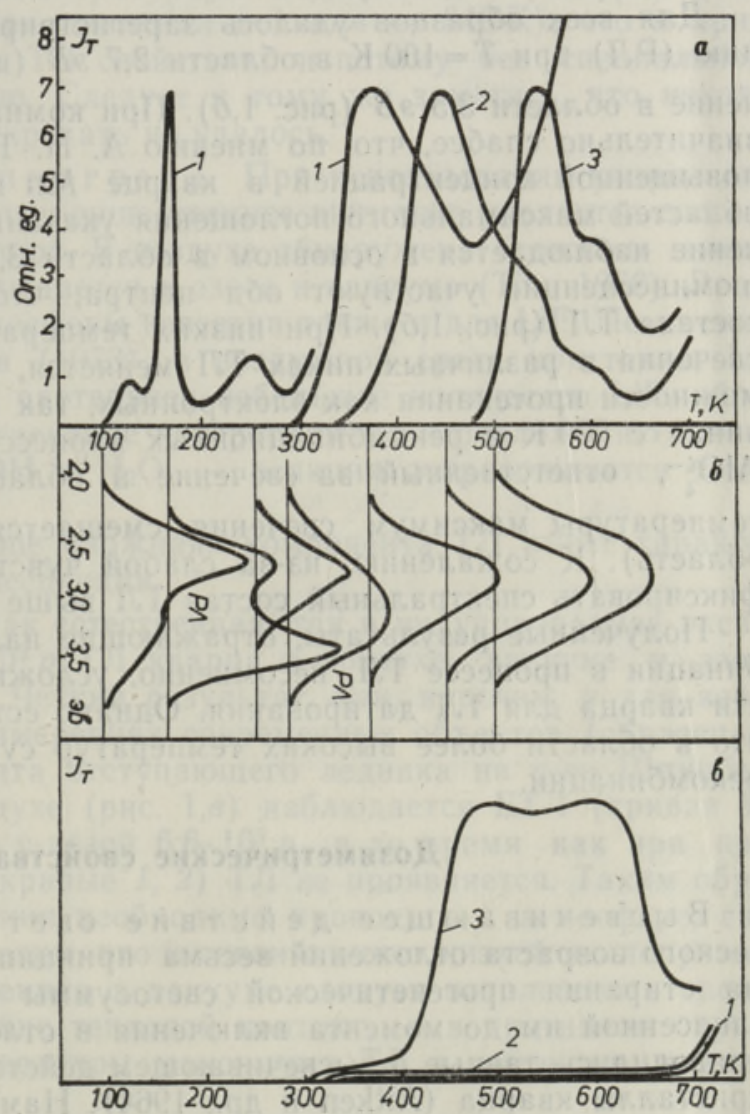


Нами было исследовано около 20 образцов кварца из осадочных отложений Эстонии различного типа и генезиса.

Морфология кривых ТЛ исследованных образцов оказалась весьма сходной (рис. 1,a). При искусственном облучении наиболее четко проявляется система мелких уровней захвата. Глубокие уровни, явно существующие в кварце, проявляются в виде вытянутого хвоста в высокотемпературной области (кривые 1, 2, фоновое свечение обрезалось жидкостным фильтром $\mathrm{CuSO}_{4}$ ). Система глубоких уровней захвата отражена на кривой ЕТЛ (3).

Кривые ТЛ для разных образцов различаются, в основном, лишь интенсивностями максимумов свечения, а также соотношениями интенсивностей отдельных пиков. Эти особенности связаны не с изменениями структуры дефектов, обусловливающих появление тех или иных пиков кривых ТЛ, а с различной плотностью дефектов кристаллической решетки (с числом дефектов на $1 \mathrm{~cm}^{3}$ кристалла). Однотипность морфологии кривых ТЛ (а следовательно, и сходство дефектности кристаллической решетки) различных образцов можно объяснить целым рядом шричин. Наиболее вероятной из них является та, что объектом исследования служил кварц, претерпевший существенные изменения в процессе транспортировки с места сноса.

Общность ТЛ свойств кварца из различных отложений - явление весьма благоприятное для использования его в качестве дозиметра, ибо это дает основание предполагать, что светосуммы, запасенные на ловушках, обусловлены одинаковыми структурными дефектами и, следовательно, сравнимы.

Для всех образцов удалось зарегистрировать рентгенолюминесценцию (РЛ) при $T=100 \mathrm{~K}$ в области 2,7 эB (центр $\mathrm{AlO}_{4}^{4-}$ ) и слабое свечение в области 3,5 эB (рис. 1,б). При комнатной температуре свечение значительно слабее, что, по мнению А. Н. Таращана (1974), связано с повышенной концентрацией в кварце $\mathrm{Mn}$ или $\mathrm{Fe}(2,7$ эB - одна из областей максимального поглощения указанных ионов). При $290 \mathrm{~K}$ свечение наблюдается в основном в области 3,5 эB. В рекомбинационной люминесценции участвуют оба центра, что видно из спектральното состава ТЛ (рис. 1,б). При низких температурах спектральный состав свечения в различных пиках ТЛ меняется, что свидетельствует о возможности протекания как электронных, так и дырочных процессов. Начиная с $200 \mathrm{~K}$ в рекомбинационных процессах участвует лишь центр $\mathrm{AlO}_{4}^{4-}$, ответственный за свечение в области 2,8 эB (с повышением температуры максимум свечения смещается в высокоэнергетическую сбласть). К сожалению, из-за слабой чувствительности установки зафиксировать спектральный состав ТЛ выше $500 \mathrm{~K}$ нам не удалось.

Полученные результаты, отражающие наличие двух каналов рекомбинации в процессе ТЛ, несомненно, усложняют проблему применимости кварца для ТЛ датирования. Однако есть основания предполагать, что в области более высоких температур существует лишь один центр рекомбинации.

\section{Дозиметрические свойства кварца}

Высвечи в ющее де й стви е света. При определении физического возраста отложений весьма принципиальной является проблема стирания прогенетической светосуммы кварца, т. е. светосуммы, запасенной им до момента включения в отложения. В литературе уже приводились данные о высвечивающем действии света на возбужденные кристаллы кварца (Aitken и др., 1964). Нами было установлено, что в 
течение 3 и воздействие УФ-света (лампа ОИ-18 с фильтром УФС-2) снижает запасенную светосумму кварцевого образца, возбужденного $\gamma$-источником ${ }^{60} \mathrm{Co}$ экспозиционной дозой $10^{5} p$, до $12 \%$ на уровне захвата, обусловливающем пик с $T_{M}=540 \mathrm{~K}$. Воздействие УФ-света такого же спектрального состава на девозбужденный образец индуцирует лишь слабый пик в области $T_{\text {M }}=430 \mathrm{~K}$. ЕТЛ экстрагированного кварца под действием обычного рассеянного дневного света $\left(10^{2} \wedge \kappa\right)$ также существенно меняется: за трое суток хранения на свету образец теряет до $50 \%$ запасенной светосуммы, а при воздействии прямого солнечного света $\left(10^{5} л \kappa\right)$ - гораздо больше.

Влияние измельчения кварца на запасенную светосумму в литературе освещено противоречиво (Seeley, 1975), вероятно, в связи с тем, что при некоторых способах измельчения возникает триболюминесценция. искажающая результаты.

Рядом авторов (Tite, 1966; и др.) наблюдалась сенсибилизация кварца, т. е. увеличение чувствительности после дрейнирования образца при температуре $670 \mathrm{~K}$ и повторного воздействия дозами $10^{3} p$. Такое явление, известное, например, и для $\mathrm{LiF}$ (Франк, Штольц, 1973), связано с существованием более глубоких уровней захвата, не опустошающихся при данной температуре прогрева. При неоднократном использовании кварцевого образца с целью получения воспроизводимых результатов (доза $\sim 10^{3} p$ ) нами было установлено, что для дрейнирования достаточен отжиг при $T=830 \mathrm{~K}$ в течение 40 мин. Использование более высоких температур может вызвать переход низкотемпературной модификации $\alpha$-кварца в высокотемпературную модификацию $\beta$-кварца (температура $\alpha$-, $\beta$-перехода при давлении в 1 aтм $-846 \mathrm{~K})$, что в принципе может отразиться на ТЛ свойствах, и потому без специального исследования нежелательно. Следует к тому же заметить, что некоторые образцы кварца дрейнировать не удалось.

Роль атм осферы прогрева. При использовании кварца для ТЛ датирования в археологии существенное внимание уделяется составу атмосферы прогрева образцов. В воздухе обнаружена «ложная» компонента ТЛ, которая не наблюдается в азоте и вакууме (Tite, 1966). Зависимость ТЛ от состава атмосферы известна также и для $\mathrm{LiF}$. По мнению M. Франка и В. Штольца (1973), в воздушной среде кристаллы LiF сорбируют влагу, которая растворяет небольшое количество $\mathrm{LiF}$, образуя гидрат $\mathrm{LiOH} \cdot \mathrm{H}_{2} \mathrm{O}$, нагревание которого ведет к разложению его на исходные компоненты $\mathrm{LiOH}$ и $\mathrm{H}_{2} \mathrm{O}$, - реакция сопровождается хемилюминесценцией.

По-видимому, и в кварце «ложная» компонента ТЛ имеет характер поверхностной хемилюминесценции.

Нами была измерена как естественная, так и индуцированная экспозиционными $\gamma$-дозами $>10^{3} p$ ТЛ кварца в воздухе, вакууме и азоте высокой степени чистоты. Четкий результат, как, впрочем, и для керамики, был получен при измерениях современных объектов (образец из озовых отложений у фронта отступающего ледника на о-ве Шпицберген). При прогреве в воздухе (рис. 1,8) наблюдается ЕТЛ (кривая 3), эквивалентная облучению $\gamma$-дозой $6,6 \cdot 10^{3} p$, в то время как при прогреве в азоте и вакууме (кривые 1,2$)$ ТЛ не проявляется. Таким образом, возрастные определения необходимо проводить в атмосфере, свободной от кислорода. Заметим, что в связи с очень низкой теплопроводностью кварца при измерениях в вакууме следует использовать специальные смазки, улучшающие тепловой контакт, - в противном случае возможно смещение температуры максимума ТЛ в высокотемпературную область. 
3 ависимость ТЛ кварца от поглощенной дозы. Известно, что кварц из древней керамики (Seeley, 1975) обладает линейной зависимостью ТЛ от дозы до $5 \cdot 10^{3}$ paд, со стадией нелинейности до $\sim 300$ рад (рис. 2,a). Такая двустадийность связана, по-видимому, с тем, что на первой стадии облучения создается некоторая дополнительная часть радиационных дефектов, на второй - происходит лишь заполнение имеющихся ловушек. Нами была исследована зависимость ТЛ от поглощенной дозы в области $2 \cdot 10^{3}-10^{6}$ рад (в связи с недостаточной чувствительностью аппаратуры измерить ТЛ для доз, меньших $2 \cdot 10^{3}$ рад, не удалось). В качестве источников возбуждения использовались $\gamma$-источники ${ }^{60} \mathrm{Co}$, мощности экспозиционных доз составляли 1,5 и $0,1 \mathrm{p} / \mathrm{ce \kappa}$.

Для кварца из разнотипных отложений получены весьма сходные данные, свидетельствующие о том, что для обеих мощностей доз область линейности простирается вплоть до $5 \cdot 10^{6}$ рад (рис. $2, a-$ сплошная линия). Образцы различались лишь чувствительностью. По оценкам С. Флеминга и Д. Стоунгама (Fleming, Stoneham, 1971), средняя годичная $(\beta+\gamma)$-доза для археологических захоронений на территории Англии

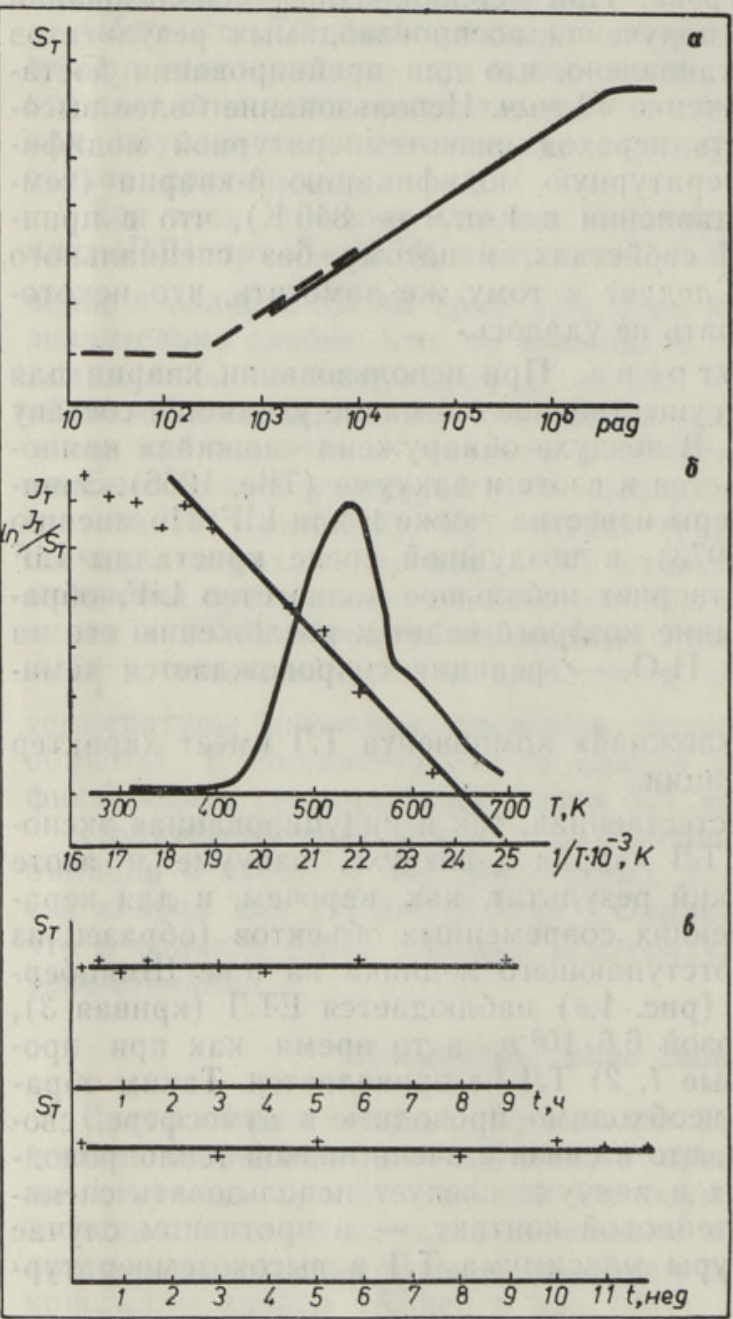
не превышает 1 рад, что дает возможность оценить верхний предел датирования приблизительно в $5 \cdot 10^{6}$ лет.

Сохранение 3 а пасенной светосуммы на глубоких уровня х захват а весьма важная проблема при использовании кварца для ТЛ датирования. Как известно, существует целый ряд каналов утечки информации, учет которых является в общем случае недостижимой экспериментальной задачей.

В случае существования лишь тепловых выбросов носителей заряда с уровня захвата в зону проводимости (тепловой фединг), время сохра-

Рис. 2. Дозиметрические свойства кварца: $a-3$ звисимость запасенной светосуммы от поглощенной дозы (пунктирная линия - по данным М.-А. Сиили (Seeley, 1975)); б- зависимость $\ln I_{T} / S_{T}, 1 / T$ для пика с $T_{M}=540 \mathrm{~K} ; 8-$ зависимость сохранения запасенной светосуммы на уровне захвата, обусловливающего пик ТЛ с $T_{M}=540 \mathrm{~K}$, от времени (температура хранения - комнатная). 
нения электронов или дырок на данном уровне захвата определяется формулой

$$
1 / t=P_{0} \mathrm{e}^{-E / k T},
$$

где $t-$ время жизни носителей на данном уровне захвата, $P_{0}-$ частотный фактор, $E-$ энергетическая глубина ловушки, $T-$ температура хранения, $k$ - константа Больцмана.

Определение тепловых энергий выброса $(E)$ с различных центров захвата требует предварительного определения типа кинетики электронно-дырочных процессов. С этой целью мы исследовали пик ЕТЛ при $T_{M}=540 \mathrm{~K}$ (наименее глубокий уровень захвата, проявляемый в ЕТЛ) по методу, предложенному В. В. Антоновым-Романовским (1946). На рис. 2,б показана выпрямленная кривая ТЛ в координатах $\ln I_{T} / S_{T} ; 1 / T$, где $S_{T}=\int_{T}^{\infty} I d T$ (I $I_{T}-$ интенсивность ТЛ и $S_{T}-$ запасенная светосумма).

Прямая в этих координатах - признак квазимономолекулярности процессов. Видно, что пик неэлементарен в области высоких температур. Из наклона участка прямой была вычислена величина энергии тепловой активации $E=1,2$ эB. Та же величина получается при оценке энергетической глубины ловушки по расчетной формуле, предложенной Ч. Б. Лущиком (1955):

$$
E=\frac{2 k T_{M}^{2}}{\delta_{m}}
$$

где $T_{M}$ - температура максимума ТЛ, $\delta_{m}$ - полуширина пика ТЛ.

Для частотного фактора мы воспользовались величиной, полученной английскими исследователями (Aitken и др., 1974) для соответствующего уровня захвата $P_{0} \approx 10^{8}$ сек ${ }^{-1}$. Расчет по формуле (1) с использованием полученных значений $E$ и $P_{0}$ показывает, что при $290 \mathrm{~K}$ (в действительности среднегодичная температура на средних широтах ниже) сохранение светосуммы обеспечивается до $10^{6}$ лет. Таким образом, термический фединг исключается. Возможен еще и аномальный фединг, т. е. фединг, связанный, например, с такими каналами потери светосуммы, как донор-акцепторные (межпримесные) переходы. Нами было проверено сохранение запасенной светосуммы на уровне захвата с $T_{M}=540 \mathrm{~K}$ за период от 10 мин после возбуждения до 3 месяцев (рис. 2,8 ). Потерь светосуммы обнаружено не было.

К такому же выводу пришли У. Ольдред и С. Флеминг (Aldred, Fleming, 1974) при изучении кварца из древней керамики. Они нашли, однако, что у полевопо шпата аномальный фединг за 2 месяца в отдельных случаях достигал $40 \%$ с таких же глубоких уровней захвата, что и у кварца.

Результаты исследования свидетельствуют о том, что при наличии высокочувствительной аппаратуры и атмосферы прогрева, свободной от кислорода, чистый кварц может быть применен для ТЛ датирования в геологических интервалах времени $10^{3}-5 \cdot 10^{6}$ лет.

Однако окончательный ответ на вопрос о степени стирания прогенетической светосуммы кварца и фединга в течение актуальных в геологии временных интервалов может быть дан лишь на основании проверкі результатов конкретной ТЛ методики датирования на реперных образ- 
цах, возраст которых установлен другими методами датирования и геологическими данными.

В заключение авторы приносят благодарность Я.-М. Пуннингу за обсуждения и Я. Луттю за консультации по геологическим проблемам.

\section{Л И ТЕ РА Т У РА}

Ан тонов - Р ом анов в кий В. В. 1946. О рекомбинационной фосфоресценции. Изв. АН СССР, сер. физ., 10, № 3 .

Б а т р а к Е. Н. 1958. Кристаллография, т. 3, вып. 5. М.

Данн и льс Ф., Бойд Ч., С а унде р с Д. 1953. Термолюминесценция как средство научного исследования. УФН, 51 , вып, 2.

Д эн а Дж. Д., Дэн а Э. С., Фр онде ль К. 1966. Система минералогии, т. 3, М.

З аки с Ю. Р., Т рухин А. Н., Хи м о в В. П. 1973. Элементарные электронные возбуждения в кварце. ФТТ, 15 , № 1 .

К ац М. Я., С и м анов и ч И. М. 1974. Кварц кристаллических горных пород. М.

Л ущи к Ч. Б. 1955. Исследование центров захвата в щелочногалоидных кристаллофосфорах, Тр. ИФА АН ЭССР, № 3.

Менд зи н я А. С., Т а л е И. А. 1974. Энергетический спектр ловушек в кристаллическом и стеклообразном кварце. Тезисы докл. III Всесоюз. симп. «Оптические и спектральные свойства стекол». Л.

Т а р а щ а н А. Н. 1974. Исследование люминесценции и термолюминесценции минералов. Автореф. докт. дис. М.

Физические исследования кварца, под ред. Самойловича М. И. 1975. М.

$\Phi$ ранк М., Ш тольц В. 1973. Твердотельная дозиметрия ионизирующего излучения. $M$.

Х ю т т Г. И., Р а у к а с А. В. 1977. О перспективах использования термолюминесцентного метода для определения возраста четвертичных отложений. Бюл. Ком. пз изучению четвертичного периода, № 47. М.

Шелкопля с В. Н. 1974. Термолюминесцентный метод и его применение для стратиграфии плейстоценовых субаэральных отложений. Автореф. докт. дис. Киев.

Aitken, M. J., Huxtable, J., Wintle, A. G., Bowman, S. G. E. 1974. Age determination by TL. Review of Progress at Oxford. Proc. 4th Intern. Conf. on Luminescence Dosimetry. Krakow, Poland.

Aitken, M. J., Tite, M. S., Reid, J. 1964. Thermoluminescent dating of ancient ceramics. Nature, No. 202. London.

Aitken, M. J., Z immerman, D. W., Fleming, S. J. 1968. Thermoluminescent dating of ancient pottery, Nature, No. 219. London.

Aldred, Y. C., Fleming, S. J. 1974. The effect of anomalous fading and supralinearity on the thermoluminescent dating of ceramics. Nature, No. 202. London.

F1eming, S. J. 1970. Thermoluminescent dating: refinement of the quartz inclusion method. Archaeometry, No. 2.

Fleming, S. J., S toneham, D. 1971. New techniques of thermoluminescent dating of ancient pottery. Proc. 3rd Intern. Conf. on Luminescence Dosimetry. Risø, Denmark.

S e e ley, M.-A. 1975. Thermoluminescent dating in its application to archaeology. J. of Archaeol. Sci., No. 2.

Tite, M. S. 1966. Thermoluminescent dating of ancient ceramics. Archaeometry, No 9.
Институт геологии
Академии наук Эстонской ССР
Поступила в редакцию
$27 / \mathrm{V} 1976$

\section{Galina HUTT, Kai VARES, A. SMIRNOV \\ KVATERNAARSETE SETETE KVARTSI TERMOLUMINESTSENTS JA DOSIMEETRILISED OMADUSED}

Uuriti kvartsi termoluminestsentsi ja dosimeetrilisi omadusi settekivimite vanuse määramise eesmärgil.

Settekivimite koostisse kuuluvate radioaktiivsete lisandite $\mathrm{U}$, Th ja ${ }^{40} \mathrm{~K}$ kiirguse toimel akumuleerib kvarts energiat valgussumma näol. Näidatakse, et valgussumma on proportsionaalne kiirgusdoosiga kuni $10^{6} \mathrm{R}$, informatsioon säilib kasutataval haardenivool $10^{6}$ aas- 
tat. Põhjendatakse vajadust mõõta kvartsi termoluminestsentsi hapnikuvabas atmosfääris ning tuuakse mõningaid fakte, mis kinnitavad, et settimisprotsessis kvartsi varasem valgussumma kaob.

Tehakse järeldus, et sobiva metoodika ja küllalt tundliku aparatuuri korral saab settekivimeid termoluminestsentsimeetodil dateerida vahemikus $10^{3}-10^{6}$ aastat. Meetod vajab veel kontrollimist võimalikult paljude reeperproovidega.

\section{Galina HOTT, Kai VARES, A. SMIRNOV}

\section{THERMOLUMINESCENT AND DOSIMETRIC PROPERTIES OF QUARTZ FROM QUATERNARY DEPOSITS}

The present paper is an attempt to study the dosimetric and thermoluminescent peculiarities of quartz with the purpose of using it for dating sedimentary deposits.

Radiation of the radioactive impurities $\mathrm{U}$, Th, ${ }^{40} \mathrm{~K}$, comprised in sedimentary deposits, induces the accumulation of energy by quartz in the form of thermoluminescence.

It is shown that the accumulated thermoluminescence is proportional to the radiation dose up to $10^{6} \mathrm{R}$, whereas the storage of information on the actual level is guaranteed for $10^{6}$ years.

The reasons for measuring the thermoluminescence in oxygen-free atmosphere are presented, as well as some facts evidencing the quenching of the thermoluminescence stored before its inclusion into the sedimentary deposits. On the grounds of the thermoluminescence and dosimetric peculiarities of quartz it is concluded that in case of appropriate methods and sensitive apparatus the thermoluminescent method can be used for dating sedimentary deposits within an interval from $10^{3}$ to $10^{6}$ years. Stress is laid upon the necessity of checking up every method on as many samples
of known age as possible. 